\title{
On non-computability of dynamic stochastic general equilibrium
}

\author{
William Glaciel
}

\begin{abstract}
As can be seen in Richter and Wong (1999), non-computability of general equilibrium has been recognized in economics. However, despite general non-computability, equilibrium can indeed be computed in specific cases. In this paper, further restriction on computability of equilibrium is provided in contexts of dynamic stochastic general equilibrium (DSGE) models with heterogeneous agents, demonstrating that non-computability concerns apply more generally than often understood.
\end{abstract}

\section{KEYWORDS}

computability, time consistency, subgame perfection, dynamic stochastic general equilibrium, heterogeneous agent

\section{JEL CLASSIFICATION}

E13, D50

\section{Introduction}

It is well-known that general equilibrium is generally not computable even when an underlying economy behaves nicely (Richter and Wong, 1999) - both equilibrium price vector and equilibrium allocations. It must be noted, though, that this does not mean there is no economy that an equilibrium can be computed.

These computability results are often cast in static general equilibrium models, but what about dynamic general equilibrium models used in macroeconomics - DSGE models? In contrast to static general equilibrium models, DSGE models feature possibility of decision resets at each period. This necessitates time consistency (or dynamic consistency) - for example, when a DSGE model is deterministic (no uncertainties), expectation of a future variable must equal actual value of the variable. In a more game-theoretic and general view, subgame perfection is required. 
In this paper, it is demonstrated that two heterogeneous agents and one commodity are sufficient to generate non-computability issues when there exists an equilibrium, allowed by an infinite-horizon model through setting appropriate parameters (specification), that features every state (predetermined) variable (both endogenous and exogenous) being time-invariant. The only additional requirement is that a consumption Euler equation holds. Note the infinite horizon qualification - in this paper, every agent would be assumed to live infinitely.

The demonstration is simplified by an example with no endogenous state variable. In such a case, if a nearby model specification has a computable equilibrium, then it is possible to compute a non-computable equilibrium by the limit consideration - contradiction. Therefore, a model that allows for an equilibrium with every state variable being identical across time is not computable for other model specifications as well. This means that for these models, no approximation method can be guaranteed convergence to an actual equilibrium.

\section{Non-computability in DSGE models}

A utility-maximizing consumer $i$ solves the following problem in a heterogeneous agent DSGE model:

$$
\max _{C_{i, t}, B_{i, t}, . .} \sum_{t=0}^{\infty}\left(\beta_{i}\right)^{t} u_{i}\left(C_{i, t}, \ldots\right)
$$

subject to budget constraint:

$$
P_{t} C_{i, t}+. .+\frac{B_{i, t}}{1+i_{t}} \leq B_{i, t-1}+\ldots
$$

where $C_{i, t}$ is (quantity of) a consumption product, $B_{i, t}$ is government bonds, $\beta_{i}$ is time discount factor, $u_{i}$ is utility function, $P_{t}$ is price for $C_{i, t}, i_{t}$ is nominal interest rate set on government bonds.

While government bonds $B_{i, t}$ and associated $i_{t}$ are featured, they are only used to derive a consumption Euler equation. As far as a consumption Euler equation holds in an equilibrium, conclusions reached in this paper are not affected even if there is no government bond in a model. 
The same Euler equation can be derived from an intertemporal budget constraint that does not contain government bonds $B_{i, t}$ :

$$
P_{t} C_{i, t}+\frac{P_{t+1} C_{i, t+1}}{1+i_{t}}+. . \leq \ldots
$$

The consumption Euler equation derived can be stated:

$$
\beta_{i} \frac{u_{i}^{\prime}\left(C_{i, t+1}\right)}{u_{i}^{\prime}\left(C_{i, t}\right)} \frac{P_{t}}{P_{t+1}}=\frac{1}{1+i_{t}}
$$

In DSGE models without uncertainties, if an intertemporal budget constraint of Equation (3) is adopted, it is asserted that subgame perfection, or time consistency, applies such that decisions made at time $t$ should not change at $t+1$.

Now consider consumer $j$ and $k$. From Equation (4), we can obtain:

$$
\frac{u_{j}^{\prime}\left(C_{j, t}\right)}{u_{k}^{\prime}\left(C_{k, t}\right)}=\frac{\left(1-\gamma_{j k}\right)}{\gamma_{j k}}\left(\frac{\beta_{k}}{\beta_{j}}\right)^{t}
$$

where $\gamma_{j k}$ is some time-invariant constant.

Equation (5) seems initially innocuous, but it is not. When every state variable and parameter in an economy are time-invariant, a consumer is solving the same optimization problem at every time period. But Equation (5) says that this is impossible when $\beta_{j} \neq \beta_{k}$.

Nature of Equation (5) is subtle. If we are willing to ignore the fact that at every period a consumer is solving the same infinite-horizon optimization problem, there is no problem in utilizing Equation (5). Therefore, nature of the problem lies not on equilibrium existence, but on computability. The point is that because we cannot compute an equilibrium, we cannot even know that an equilibrium features time-invariant state variables.

It is possible that an equilibrium featuring time-invariant state variables simply does not exist instead. However, one can easily show that a specific economy must feature time-invariant state variables in an equilibrium, even though an equilibrium itself cannot be computed. When there is no endogenous state variable, as long as all exogeneous state variables are time-invariant, every consumer does face the same optimization problem at each period. Simple labor-only New Keynesian models (Woodford, 2003; Galí, 2015) often feature lack of 
endogeneous state variables except for government bonds, so this consideration should be uncontroversial.

The above example where there is no endogenous state variable raises the following issue. It is very easy to generate an economy where all parameters and exogenous state variables are time-invariant. Suppose this economic specification can be approached as a limit of different economic specifications. Let equilibrium $e=f(s)$, where $s$ is economic specification, with $f$ assumed to be continuous and $f$ being computable whenever $s$ is computable (Richter and Wong, 1999), except when $s$ is time-invariant. Then we can compute an equilibrium for an economy that has no computable equilibrium by taking the limit of $e$ as $s$ approaches the target economy. This is contradictory - therefore, in such a case, a model either generally features non-computability of an equilibrium regardless of parameter specifications or has equilibrium discontinuity ( $f$ is discontinuous).

\section{Conclusion}

Therefore, in constructive and computability sense, Equation (5) makes a DSGE model generally time-inconsistent, though not always. There were two factors driving this result. First, a model is confined to an infinite-horizon model. In a finite-horizon model, agents can never solve the same optimization problem at every period, because time horizon is decreasing. Second, Equation (5) features no reference to state variable, thereby making it susceptible to diagonalization strategies to proving non-computability.

The result of this paper extends non-computability results in static general equilibrium models to DSGE models - more problematic non-computability issues are to be expected in DSGE models.

The non-computability result may not affect how we use DSGE models. While an equilibrium may never be accurately approximated, we may be content with different approximate models, such as linearization models, which are still most commonly used for solving complex DSGE models. The non-computability result suggests that higher-order non-linear investigations may not really be worth much, because we can still be off the mark from an actual equilibrium, so we may be content with linear or second-order results. Whether this is the right stance to take is left to other works. 


\section{Conflicts of interest}

The author reports no conflict of interest.

\section{References}

Galí, J. 2015. Monetary Policy, Inflation, and the Business Cycle. Princeton University Press.

Richter, M. K. and K.-C. Wong 1999. Non-computability of competitive equilibrium. Economic Theory, 14(1):1-27.

Woodford, M. 2003. Interest and Prices. Princeton University Press. 